\title{
Urinary tract infections: etiology and antimicrobial susceptibility of uropathogens
}

\author{
Mario Laneve, Carmela Mazzone \\ Laboratorio Analisi Chimico Cliniche e Microbiologia, P.O. Occidentale (Mottola), AUSL TA
}

Key Words: urinary tract infections, Escherichia Coli, Staphylococcus aureus, antibiotic resistance

Infezioni delle vie urinarie: eziologia e profilo di sensibilità ai farmaci antibatterici

\section{SUMMARY}

Background:

Urinary tract infections are a serious health problem affecting millions of people each year.They are the second most common type of infection in the body. The objective of study was to determine the etiology and antimicrobial susceptibility patterns of urinary tract infections pathogens isolated in our Patology Clinic laboratory.

\section{Materials and Methods:}

During the period July 2007- July 2008,were analysed I422 urine samples. The determination of the total microbe load were acquire with an kit of the BIO-DETECTOR while the identification of germs with Apy sistem. Antibiotic susceptibility tests were assaied with the ATB UR strip.

Results:

About the total of samples analysed, 320 (22\%) had significant bacteriuria. Escherichia coli was the most common etiologic agent isolated (62\%), followed by Klebsiella ssp. (10\%), Pseudomonas aeruginosa (5,95\%) and Proteus mirabilis (5\%). Gram-positive bacteria accounted for only $7.32 \%$, with prevalence of Staphylococcus ssp $(5,32)$ and Enterococcus spp (2\%). The most effective antibiotics for Gram- were: Imipenem, Amikacin, Ceftazidime and Cefotaxim, while for Gram+ were: Minocyclin, Vancomycin and Oxacillin.

\section{Conclusion:}

Escherichia coli was the microrganism more frequently isolated between Gram negative bacteria with very susceptible to Amoxicillin. Currently, the empirical use of Cotrimoxazole and Amoxicillin is not recommended for Enterobacteriaceae. Urinary tract infections are more common in women than in men. Men are more likely to get a UTI once past the age of 65. Current data on the prevalence of multidrug resistance among urinary tract isolates should be a consideration to change the current empiric treatment of IVU.

\section{Received October 6, 2008}

Accepted December 30, 2008

\section{INTRODUZIONE}

Le infezioni delle vie urinarie (IVU) stanno assumendo sempre maggior rilievo da un punto di vista epidemiologico (ogni anno in Italia sono diagnosticati quasi due milioni di casi) (1); esse sono seconde solo a quelle dell' apparato respiratorio e rappresentano il $30-40 \%$ delle infezioni ospedaliere, essendo la forma più frequente di infezione nosocomiale $(3,5,17)$. Le IVU di origine nosocomiale sono molto spesso infezioni complicate e la loro eziologia differisce notevolmente da quella delle infezioni comunitarie non complicate coinvolgendo un ampia gamma di germi, sia Gram positivi che Gram negativi, sebbene il patogeno più frequentemente isolato rimanga l'E. coli $(12,21)$.

Tali infezioni sono interessanti anche da un punto di vista clinico e prognostico, per la frequenza con cui si manifestano e per le complicanze che da esse derivano (14). Le IVU possono riguardare le basse o le alte vie urinarie; i sintomi delle IVU sono quanto mai vari, potendo decorrere in maniera completamente silente (batteriuria asintomatica) o, nella loro massima espressività e gravità, manifestarsi con un quadro di pielonefrite acuta (2).

Per molto tempo i farmaci di prima scelta impiegati in queste patologie sono stati Amoxicillina, Cotrimoxazolo, Nitrofurantoina e Fluorochinoloni $(4,8,9,13,15)$, ma come vedremo in seguito alcuni di questi antibiotici sono attualmente inefficaci. Questo studio si propone di valutare la frequenza delle IVU nei pazienti ambulatoriali esterni, i microrganismi coinvolti e la sensibilità dei germi

\section{Corresponding author: Mario Laneve}

Laboratorio Analisi Chimico Cliniche, P.O.: Occidentale (Mottola)

Via Nitti n. I -74100 Taranto, Italy - Tel: 3477204784

E-mail: mario.laneve@yahoo.it 
nei confronti dei chemioterapici di più largo impiego in terapia.

\section{MATERIALI E METODI}

Nel periodo compreso tra giugno 2007 e giugno 2008, presso il Laboratorio di Patologia Clinica del P.O. Occidentale (Mottola) della AUSL Taranto, sono state esaminate 1422 urinocolture provenienti da pazienti. ambulatoriali esterni con sospetto di infezione del tratto urinario.

La determinazione della carica batterica è stata ottenuta mediante il kit bio-detector (Liofilchem), che utilizza dei dip-slide. Lo slide formato da un supporto di plastica ospita 5 terreni colturali: il terreno CLED Agar (di colore verde) che consente la determinazione della carica microbica totale, il terreno MacConkey Agar (di colore rosso-viola) per la crescita dei batteri Gram negativi, in particolare gli Enterobatteri, il terreno Cetrimide Agar (di colore ambra chiaro) per la crescita dei batteri del genere Pseudomonas, il terreno Bile Aesculin Agar (di colore ambra) per gli Enterococchi e il terreno Malto Agar (di colore ambra scuro) per i miceti.

La modalità di raccolta delle urine è avvenuta mediante il mitto intermedio, che consiste nel raccogliere le urine dalla prima minzione del mattino dopo un soggiorno in vescica di almeno 5 ore.

Per ottenere un idoneo materiale da un paziente in grado di cooperare è sufficiente far osservare alcuni accorgimenti tra cui, un lavaggio accurato dei genitali e, dopo aver scartato il primo getto si raccoglie la rimanente urina in un contenitore sterile. Lo slide viene immerso nel campione d'urina e incubato in stazione termostatata a $37^{\circ} \mathrm{C}$ per 24 ore. Dopo incubazione la presenza di batteri è evidenziata dalla comparsa di colonie sulle superfici dei terreni colturali. L'urinocoltura è considerata positiva per una carica uguale o superiore a $10^{5}$ $\mathrm{CFU} / \mathrm{ml}$. Tuttavia, in presenza di significativa sintomatologia (dati clinici forniti dai medici di medicina generale e di reparto) e in base all'esame chimico fisico delle urine, sono state prese in considerazione anche cariche batteriche più basse. Batteri Gram-, come Escherichia Coli o Klebsiella spp, una volta cresciuti sul terreno MacConkey Agar, vengono poi identificati mediante test biochimici.

L'identificazione dei germi Gram- e Gram+ è stata ottenuta con i sistemi API 20 E, API Strep, API Staph (bioMèrieux), che si basano sull'impiego di prove biochimiche.

I relativi antibiogrammi sono stati ottenuti mediante i kit $\mathrm{ATB}^{\mathrm{TM}} \mathrm{UR} 5$, $\mathrm{ATB}^{\mathrm{TM}}$ PSE, $\mathrm{ATB}^{\mathrm{TM}}$ STAPH 5, ATB ${ }^{\text {TM }}$ STREP 5 (bioMèrieux).

Le gallerie ATB UR sono costituite da diverse coppie di cupole. La prima coppia, senza antibiotico, costituisce il controllo di crescita. Le altre successive contengono antibiotici ad un'unica o due con- centrazioni (c e C). Il batterio da saggiare viene messo in sospensione (API Suspension Medium) e quindi trasferito nel terreno di coltura MullerHinton (ATB Medium) ed inoculato nella galleria. La quantità di sospensione batterica di partenza e il tipo di ATB Medium cambiano in base al germe isolato. Dopo l'incubazione $\left(37^{\circ} \mathrm{C}\right.$ per 24 ore), la lettura della crescita batterica viene eseguita visivamente. La presenza di torbidità $(+)$ in una o entrambe le cupole per ogni antibiotico, consente di classificare il ceppo come intermedio o resistente; l'assenza di torbidità (-) in entrambe le cupole classifica il ceppo come sensibile. La sensibilità agli antibiotici è stata determinata mediante breakpoints cioè concentrazioni definite come soglia per esprimere la sensibilità e la resistenza dei microrganismi agli antibiotici. L'identificazione dei lieviti è stata eseguita con API 20 C AUX (bioMèrieux) e l'antimicogramma con l'ATB ${ }^{\mathrm{TM}}$ FUNGUS 3 (bioMérieux). I risultati sono stati valutati in base alle indicazioni riportate da CLSI (21).

\section{RISULTATI E DISCUSSIONE}

Delle 1422 urinocolture analizzate, 320 sono risultate positive (22\%), con prevalenza del sesso femminile (75\%) rispetto al sesso maschile (25\%), questo a causa della conformazione dei genitali esterni e la brevità dell'uretra nella donna (Figura I e II).

L'incidenza delle IVU aumenta con l'età; nella donna, è molto bassa nell'età prepuberale mentre con l'inizio dell'attività sessuale e le gravidanze aumenta, e continua ad aumentare anche dopo la menopausa; nell'uomo tende ad aumentare in età avanzata (intorno ai 65 anni), specialmente a causa di ipertrofia prostatica.

La maggior parte delle IVU sono sostenute da batteri Gram negativi $(92.05 \%)$ con una prevalenza di Escherichia coli (62\%), seguita da Klebsiella pneumoniae (7.5\%), Pseudomonas aeruginosa (5.95\%) e Proteus mirabilis (5\%).

Complessivamente, le Enterobacteriaceae costitui-

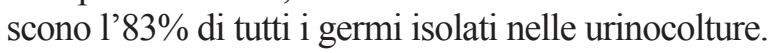
Tra i Gram positivi (7.32\%) prevalgono gli Stafilococchi: S. aureus (3.75\%), S. saprophyticus $(0.94 \%)$ e $S$. haemolyticus $(0.63 \%)$, e gli Enterococchi spp (2\%). Poco rilevante è la presenza di miceti con solo lo $0.63 \%$ di Candida albicans. In basse percentuali si presentano tutti gli altri germi mostrati nella Tabella 1. Le percentuali di antibioticosensibilità dei batteri Gram negativi più frequentemente isolati sono riportate nella Tabella 2, mentre le percentuale di antibiotico-sensibilità dei batteri Gram positivi sono riportate in Tabella 3. Dai risultati delle nostre osservazioni si evidenzia che Amoxicillina (AMO) e Cotrimoxazolo (TSU), farmaci comunemente usati per il trattamento delle IVU, presentano alte percentuali di resistenza nei confronti dei più 


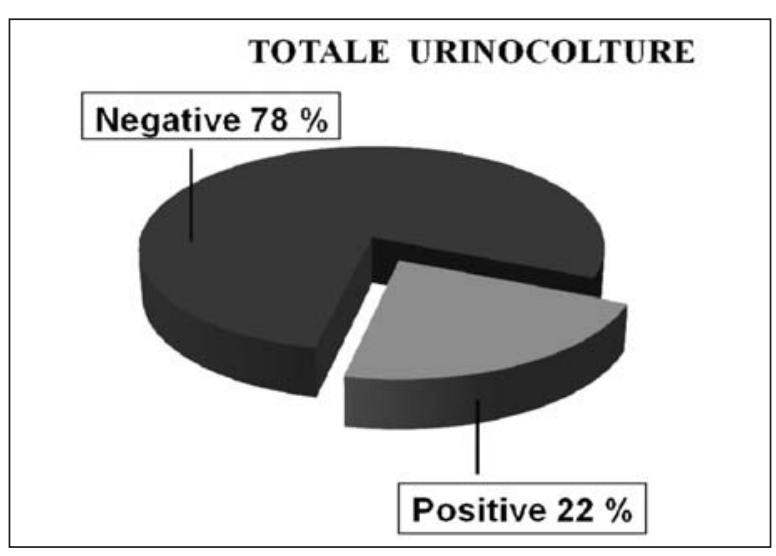

Figura I. Percentuali di positività delle urinocolture

comuni germi Gram negativi (mediamente 59\% per AMO e $60.5 \%$ per TSU), mentre nei confronti dei Gram positivi, l'AMO presenta resistenza intorno al $70 \%$ e il TSU intorno al $25 \%$.

Associando però l'Amoxicillina all'acido clavulanico, la resistenza da parte dei Gram negativi si riduce (22\%), ma non per Pseudomonas aeruginosa e batteri Gram positivi.

Imipenem, Fosfomicina, Amikacina,Ceftazidima, Cefotaxima e Gentamicina (quest'ultimo tranne per Proteus spp.), sono risultati in media i più attivi nei confronti dei Gram negativi, con una sensibilità superiore al 70\%; mentre Minociclina, Vancomicina e Oxacillina si sono rivelati molto efficaci nelle infezioni da stafilococchi, con un alta percentuale di sensibilità pari al 99\%.

L'Imipenem si è distinto come il farmaco maggiormente attivo nei confronti dello Pseudomonas aeruginosa. con percentuali di sensibilità del $75 \%$, seguita dalla Piperacillina con il 70\%.

Per quanto riguarda gli enterococchi, solo la Fosfomicina ha mostrato la più elevata sensibilità, con il 56\%, tutti gli atri antibiotici si sono rilevati meno attivi. In particolare la Nitrofurantoina è

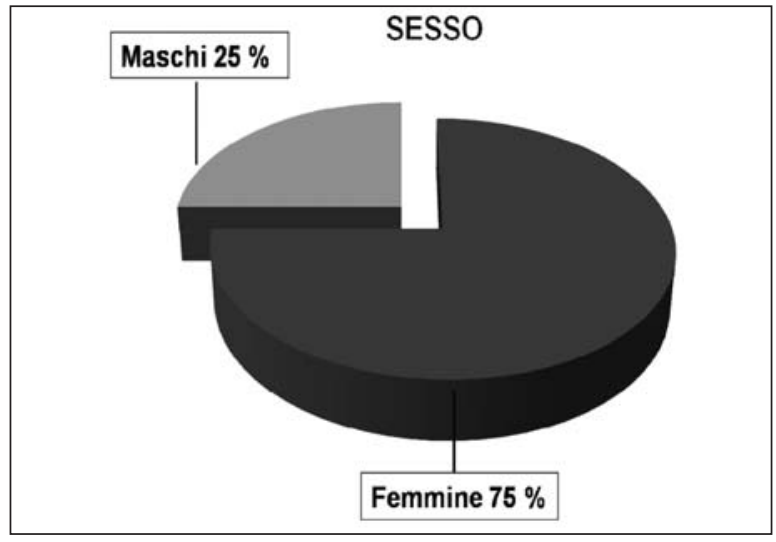

Figura II. Distribuzione delle urinocolture positive correlate al sesso

stato l'antibiotico che ha mostrato una diffusa resistenza pari al $91 \%$.

Tabella I. Ceppi batterici isolati nei pazienti ambulatoriali

\begin{tabular}{lcc}
\hline $\begin{array}{l}\text { Microrganismi } \\
\text { identificati }\end{array}$ & $\mathbf{n}^{\circ}$ ceppi & $\begin{array}{c}\text { \% sul totale } \\
\text { degli isolati }\end{array}$ \\
\hline Escherichia Coli & 198 & $62 \%$ \\
\hline Klebsiella pneumoniae & 24 & $7,5 \%$ \\
\hline Pseudomonas aeruginosa & 19 & $5,95 \%$ \\
\hline Proteus mirabilis & 16 & $5 \%$ \\
\hline Staphylococcus aureus & 12 & $3,75 \%$ \\
\hline Citrobacter freundii & 8 & $2,5 \%$ \\
\hline Serratia odorifera & 8 & $2,5 \%$ \\
\hline Enterococcus spp. & 7 & $2 \%$ \\
\hline Morganella morganii & 6 & $1,89 \%$ \\
\hline Aereomonas sobria & 4 & $1,25 \%$ \\
\hline Klebsiella ornithinolytica & 4 & $1,25 \%$ \\
\hline Staphylococcus saprophyticus & 3 & $0,94 \%$ \\
\hline Staphylococcus haemolyticus & 2 & $0,63 \%$ \\
\hline Klebsiella Planticola & 2 & $0,63 \%$ \\
\hline Candida albicans & 2 & $0,63 \%$ \\
\hline Klebsiella Oxytoca & 2 & $0,63 \%$ \\
\hline Enterobacter cloacae & 2 & $0,63 \%$ \\
\hline Citrobacter Koseri & 1 & $0,32 \%$ \\
\hline
\end{tabular}

Tabella 2. Percentuali di antibiotico-sensibilità dei batteri Gram negativi di più frequente riscontro

\begin{tabular}{lcccccccccccc}
\hline Microrganismo & AM & AM & PIC & CIP & TS & CA & CT & GE & IMI & AK & FO & FU \\
& O & C & & & U & I & $\mathbf{X}$ & $\mathbf{N}$ & & $\mathbf{N}$ & S & R \\
\hline E. coli & 44 & 85 & 68 & 78 & 56 & 94 & 96 & 87 & 100 & 99 & 100 & 99 \\
\hline Klebsiella spp & 55 & 70 & 86 & 57 & 52 & 86 & 86 & 86 & 57 & 86 & 86 & 86 \\
\hline Proteus spp & 45 & 80 & 75 & 45 & 25 & 67 & 71 & 18 & 75 & 75 & 83 & 17 \\
\hline Pseudomonas a. & 20 & 20 & 70 & 45 & 25 & 68 & 69 & 60 & 75 & 70 & $/$ & $/$ \\
\hline
\end{tabular}

Legenda: Amoxicillina (AMO), Amo-ac.cla (AMC), Piperacillina (PIC), Ciprofloxacina (CIP), Cotrimoxazolo (TSU), Ceftazidima (CAI), Cefotaxima (CTX), Gentamicina (GEN), Imipenem (IMI), Amikacina (AKN), Fosfomicina (FOS), Nitrofurantoina (FUR).

Tabella 3. Percentuali di antibiotico-sensibilità dei batteri Gram positivi

\begin{tabular}{lcccccccccc}
\hline Microrganismo & $\begin{array}{c}\text { AM } \\
\text { O }\end{array}$ & AMC & CIP & TSU GEN & FOS & FUR & MIN & VAN & OX \\
Staphylococcus sp & 27 & 30 & 45 & 75 & 70 & 50 & 65 & 100 & 100 & 99 \\
\hline Enterococcus spp & 28 & 30 & 40 & $/$ & 20 & 56 & 9 & $/$ & $/$ & $/$ \\
\hline
\end{tabular}

Legenda: Amoxicillina (AMO), Amo-ac.cla (AMC), Ciprofloxacina (CIP), Cotrimoxazolo (TSU), Gentamicina (GEN), Fosfomicina (FOS), Nitrofurantoina (FUR), Minociclina (MIN), Vancomicina (VAN), Oxacillina (OXA). 


\section{CONCLUSIONI}

I risultati di questa indagine indicano che 1'Escherichia coli è il batterio più frequentemente isolato (62\%) tra i batteri Gram negativi responsabili delle IVU, analogamente a quanto riportato in letteratura $(11,18,19,20)$ e la variabilità dei germi responsabili di tali infezioni aumenta sempre più (10). La positività dei campioni è più elevata nei pazienti di sesso femminile (75\%) e l'incidenza delle IVU aumenta con l'età. La resistenza dell' $E$. coli nei confronti delle principali classi di farmaci impiegati in terapia, si è mostrata elevata per l'Amoxicillina e Cotrimoxazolo (in media il 50\%), così come per le altre Enterobacteriaceae. Solamente Imipenem, Amikacina, Fosfomicina e Nitrofurantoina hanno mostrato percentuali di sensibilità intorno al 99\%. La multi-resistenza antibiotica riscontrata tra $i$ batteri Gram negativi è ormai un fenomeno comune, prodotta spesso da un uso incongruo della terapia antibiotica.

Nell'ambito dei batteri Gram positivi prevalgono gli stafilococchi (5.32\% di tutti i germi responsabili).

Tra gli antibiotici, l'Amoxicillina ha mostrato molta resistenza (superiore al 70\%), mentre il Cotrimoxazolo è risultato dotato di una convincente attività antibatterica.

Minociclina, Vancomicina e Oxacillina, si sono distinti come i farmaci maggiormente attivi sugli stafilococchi. Le infezioni complicate del tratto urinario stanno, dunque, diventando un problema sempre più serio, e la diffusione delle resistenze nei confronti dei farmaci di prima scelta contro le IVU, come AMO e TSU utilizzati per diversi anni, porterebbe a una rivalutazione della terapia antibiotica. Occorre, dunque, sottolineare la fondamentale importanza di indicare un'adeguata e corretta terapia farmacologica, sia per risolvere rapidamente ed efficacemente l'infezione, sia per prevenire la selezione e la diffusione dell'antibiotico resistenza nei microrganismi $(6,16)$.

Studi come questo possono fornire al clinico informazioni preziose sull'incidenza locale di resistenza agli antibiotici più utilizzati nelle IVU. I risultati di queste osservazioni confermano l'utilità del laboratorio di patologia clinica nella sorveglianza delle IVU

\section{BIBLIOGRAFIA}

1. Alos JI. Epidemiology and etiology of urinary tract infections in the community. Antimicrobial susceptibility of the main pathogens and clinical significance of resistance. Enferm Infecc Microbiol Clin. 2005; 23 Suppl 4: $3-8$

2. Anna M. Molina Romanzi. Testo di Microbiologia Clinica. "Gli aspetti microbiologici delle malattie infettive". UTET 2004; 9:186 - 06

3. Bagshaw SM, Laupland KB. Epidemiology of intensive care unit-acquired urinary tract infections.Curr
Opin Infect Dis. 2006; 19: 67 - 71

4. Barisic Z, Borzic E, Kraljevic KS, et al. Rise in ciprofloxacin resistance in Escherichia coli from urinary tract infections from 1999-2004. Int J Antimicrob Agents. 2005; 25: 550 - 551

5. Brook I. Urinary tract and genito-urinary suppurative infections due to anaerobic bacteria. Int J Urol. 2004; $3,133-41$

6. Canbaz S, Peksen Y, Tevfik Sunter A, et al. Antibiotic prescribing and urinary tract infection. Int $J$ Antimicrob Agents. 2002; 6:407 - 11

7. Clinical and Laboratory Standards Institute/NCCLS. Performance Standards for Antimicrobial susceptibility testing; fifteenth International Supplement. CLSI/NCCLS document M100 - S15. Clinical and Laboratory Standards Institute, Wayne, Pennsylvania, 2005

8. Erb A, Stürmer T, Marre R, et al. Prevalence of antibiotic resistance in Escherichia coli: overview of geographical, temporal, and methodological variations. Eur J Clin Microbiol Infect Dis. 2007; 26(2):83 - 90

9. Fadda G, Nicoletti G, Schito GC, et al. Antimicrobial susceptibility patterns of contemporary pathogens from uncomplicated urinary tract infections isolated in a multicenter Italian survey: possible impact on guidelines. J Chemother. 2005; 17:251 - 7

10. Faggi E, Farina C, Manso E, et al. Fungurie nei pazienti ospedalizzati: indagine retrospettiva multicentrica. Microbiologia Medica. 2004; 4:389 - 392

11. Farrell DJ, Morrissey I, De Rubeis D, et al. A UK multicentre study of the antimicrobial susceptibility of bacterial pathogens causing urinary tract infection. $J$ Infect 2003; 46(2):94 - 100

12. Junquera S, Loza E, Baquero F. Changes in the antimicrobial susceptibility of Escherichia coli isolates from nosocomial versus community-acquired urinary tract infections. Enferm Infecc Microbiol Clin. 2005; 23: $197-201$

13. Maioli E, DeMandina I. Antimicrobiol susceptibility survey of pathogens isolated from selected patients in Northern Italy. Microbiologia Medica 2004; 20, 12 - 21.

14. Neal DE Jr. Complicated urinary tract infections. Urol Clin North Am. 2008; 1:13 - 22

15. Nicolle LE. Resistant pathogens in urinary tract infections. J Am Geriatr Soc. 2002; 50(7 Suppl):S230

16. Ochoa Sangrador C, Brezmes Raposo M; Grupo Investigador del Proyecto. Recommended antibiotic therapy in episodes of urinary tract infection. An Pediatr (Barc) 2007; 5:485 - 97

17. Pistarà $\mathrm{P}$, Mannelli S, Marchese A, et al. Resistenza ai fluorochinoloni in Escherichia coli isolati da infezioni delle vie urinarie (IVU) in pazienti ospedalizzati in unità di terapia intensiva (UTI). Microbiologia Medica. 2007; Vol22(II): 101 - 04

18. Ronca A, Brenci S, Santoriello L, et al. Indagine epidemiologica locale dell'eziologia delle infezioni delle vie urinarie (IVU) nosocomiali e comunitarie e dell'antibiotico-sensibilità degli uropatogeni. Microbiologia Medica. 2007; Vol22 (I): 8 - 11

19. Speciale AM, Nicoletti G, Torrisi C, et al. Prevalenza e antibiotico-resistenza dei principali patogeni urinari responsabili di cistiti non complicate nella donna: lo studio ICEA 2. GIMMOC. 2004; 8 (Q1): 1 - 16

20. Tortoriello R, D’Angelo D, Martino R. Le infezioni delle vie urinarie: batteri isolati e la loro suscettibilità agli antibiotici. Patologo Clinico 2004; 1/2: 33 - 38

21. Wagenlehner FME, Naber KG. Treatment of bacterial urinary tract infections: presence and future. European Urology. 2006; 49:235 - 244 